\title{
Study of the difference of high and low metastasis cell line's gene expression map and metastasis-related genes of adenoid cystic carcinoma
}

\author{
NaiShuo Zhu ${ }^{1,3 *}$, JieLin Yang ${ }^{1 *}$, \\ YingMing Wang ${ }^{1}$ and XiaoFeng Guan ${ }^{1,2}$ \\ ${ }^{1}$ Lab of Molecular Virology \& Immunology \\ School of Life Sciences, Fudan University \\ Shanghai 200433 \\ ${ }^{2}$ The First Affiliated Hospital \\ China Medical University \\ Shenyang 110001 \\ ${ }^{3}$ Corresponding author: Tel/Fax, 86-21-6564-1215; \\ E-mail, nzhu@fudan.edu.cn \\ *These authors contributed equally to this work.
}

Accepted April 62003

Abbreviations: ACC, adenoid cystic carcinoma; CTGF, connective tissue growth factor family; G3PDH, glyceraldehyde-3-phosphate dehydrogenase; JTB, jumping translocation break-point; PTX, pertussis toxin

\begin{abstract}
We searched for metastasis-related genes in adenoid cystic carcinoma by suppression subtractive hybridization analysis of high and low metastasis cell lines. Twelve genes (ten previously identified and two novel sequences) were identified as being expressed at lower levels in high metastasis cell line Acc-M when compared to low metastasis cell line Acc-2. The known sequences corresponded to the genes for cysteine-rich angiogenesis induction factor (cyr61), chromosome 7 RP11-52501 clone, G-protein, WAS familial ferritin I heavy chain, jumping translocation breakpoint, eukaryotic translation elongation, folate receptor and three ribosomal proteins. Among them, the $G$ protein and ferritin I heavy chain genes contained mutations in the high metastasis cell line. The two novel gene sequences have been named ACC metastasis-associated RNH and ACC metastasis-associated suspected protein (GenBank \# AF522024 and AF522025, respectively). Taken together, these results suggest that reduced expression and/or mutation of several genes in the tumor cell line Acc-M are associated with high tumor metastasis, providing important molecular biological materials for further
\end{abstract}

study of metastasis control and possible targets for cancer gene therapy.

Keywords: adenoid cystic carcinoma cell line; suppression subtractive hybridization; tumor metastasis

\section{Introduction}

Metastasis and invasion are important, complex characteristics of malignant tumors (Fang et al., 2001). If a cancer loses metastatic ability, it becomes less malignant overall, making identification of tumor metastasis-related genes a necessary goal in the development of future cancer control therapies. Here, we report the use of a genetic comparison of two highand low metastasis adenoid cystic carcinoma cell lines as a strategy for identification of tumor metastasis-related genes.

Adenoid cystic carcinoma (ACC) is a commonly encountered oral-maxillofacial malignant tumor that is known to metastasize to the lung quite often, although the mechanism of this metastasis is unknown. Using metastasis in vivo continuous generation technology and in vitro clone technology, one of the authors succeeded in identifying high metastasis adenoid cystic carcinoma clone Acc-M (Guan et al., 1997a). This work was based on a study of the heterogeneity of the 162nd generation of low metastasis clone Acc-2. There is significant difference between the two cell lines: the metastasis of Acc-M is $96 \%$ whereas that of Acc-2, 18\% (Guan et al., 1997a). Since Acc-M evolved from Acc-2, the difference in metastasis rate must be conferred by genetic differences between the two, making them a good template for the research of tumor metastasis-related genes. Since the introduction of Acc-M, preliminary reports have been made on studies of cell level and phenotypic interference, e.g., difference of karyotype of low metastasis adenoid cystic carcinoma (Guan et al., 1996), tretinoin (vitamin A acid) anti-adenoid cystic carcinoma lung metastasis (Guan et al., 1997b), and so on. In order to explore the difference between the two cell lines on a molecular level, this experiment searched for genes possibly associated with tumor metastasis using suppression subtractive hybridization (SSH) and DNA sequencing technology.

Since its introduction in 1996, suppression subtrac- 
tive hybridization has been used to clone tumor related genes by comparing tumor and normal cell lines, or by comparing different tumor cell lines (VonStein et al., 1997; Guo et al., 2001). This method has been used to identify the kiss-1 gene as being closely associated with melanoma, to isolate the deme- 6 gene from a breast cancer cell line, and to isolate the rbap4p gene from Wilm's tumor (VonStein et al., 1997). At this time, no such screening of high and low metastasis cell lines of adenoid cystic carcinoma has been reported. Accordingly, we sought to examine differential gene expression and metastasis-related genes using Acc-2 and Acc-M as templates for suppression subtractive hybridization.

\section{Materials and Methods}

\section{Extraction of cell total RNA}

Acc-2 and Acc-M cell lines were purchased from Shanghai No.9 People's Hospital and maintained in RPMI 1640 (Gibco/BRL Co., Rockville, MD) supplemented with streptomycin and penicillin and 10\% FBS. The cells were harvested with $0.125 \%$ trypsin and total RNA was extracted with Trizol total RNA extraction reagent (Gibco), according to manufacturer's instructions.

\section{mRNA isolation and purification}

mRNA was purified with Oligotex ${ }^{T M}$ mRNA isolation kit (QIAGEN Co., Hilden, Germany), according to manufacturer's instructions.

\section{Suppression subtractive hybridization}

The PCR-select cDNA subtraction kit was purchased from CLONTECH (Palo Alto, CA). cDNA was reverse transcribed according to manufacturer's instructions and digested with Rsa I. Acc-2 and Acc-M were ligated with different adapters, and the ligation efficiency of the adapters was detected. Two rounds of hybridization and one PCR amplification were performed with corresponding drivers (when Acc-2 was used as tester, Acc-M was the driver and vice versa), and another two rounds of PCR were performed to obtain differential fragments. A detailed description of the protocol can be found in the CLONTECH manual.

\section{Recovery and cloning of differentially displayed fragments}

E. coli JM109 was from our lab stock. PGEMT vector was purchased from Promega Co. (San Diego, CA). After differential display, the DNA fragments were electrophoretically purified and cloned into the pGEMT vector. Positive clones were selected on X-gal/IPTG/
Amp agar plates. The G3PDH-5' primer (5'-TCCACCACCCTGTTGCTGTA-3') and G3PDH-3' (5'-ACCACAGTCCATGCCATCAC-3') primers were used to amplify the gene clone of G3PDH. The PCR primer 1 (5'-CTAATACGACTCACTATAGGGC-3') and G3PDH3 'primer (listed above) were used to amplify the clone of G3PDH that were with adapter sequence according to manufacturer's instructions (CLONTECH) for evaluation of recombinant plasmids. Comparison of G3PDH with adapter and G3PDH levels was used to determine the ligation efficiency.

\section{Sequence analysis and homologous comparison of cloned fragments}

Forty seven clones were randomly selected and sequenced by an ABI PRISM 377 sequencer in accordance with the Sanger dual-DNA sequencing method. After removing vector and nested PCR primer sequences, homologous analysis of the sequences was performed using the NCBI's BlastN program.

\section{Northern blot analysis}

Differentially expressed genes were selected, corresponding oligonucleotide probes were synthesized and Northern blot analysis of mRNA expression was performed using the RNADetector ${ }^{\mathrm{TM}}$ kit (KPL Co., Kirkegaard, and Perry Laboratories, Washington DC). Total RNA from high and low metastasis cell lines of Acc-2 and Acc-M were electrophoresed on $2 \%$ agarose formaldehyde-denaturing gels and transferred to a HybondN membrane (Amersham, Little Chalfont, Buckinghamshire, England). Probes were prepared according to manufacturer's instructions and added to the hybridization solution at a concentration of $500 \mathrm{ng}$ probe $/ \mathrm{ml}$. Membranes were allowed to hybridize overnight at $52^{\circ} \mathrm{C}$ before being washed and visualized according to manufacturer's instructions.

In addition, we used Northern blot analysis to confirm the reliability of the newly discovered gene sequences and differential expression levels. We synthesized oligonucleotide probes based on the sequence of clone C9 (AF522024, ACC metastasis-associated $\mathrm{RNH}$ ) and clone C12 (AF522025, ACC metastasisassociated suspected protein). The probe sequences were as follows. Probe C9: 5'-CTTCTACACTGCGTACACCTG-3' and probe C12: 5'-AGCGGTATCAGCTCACTCAA-3'.

\section{Reverse transcriptase-polymerase chain reaction (RT-PCR)}

Total RNA was isolated from Acc-2 and Acc-M cells with Trizol total RNA extraction reagent (Gibco), according to manufacturer's instructions. $1 \mu \mathrm{g}$ RNA was used for first strand cDNA synthesis with Oligo (dT) 
and SMART IIA oligonucleotides from the CLONTECH RACE cDNA Amplification Kit according to manufacturer's instructions. The ACC metastasis-associated $\mathrm{RNH}$ gene and ACC metastasis-associated suspected protein gene were PCR amplified with gene-specific primers (listed below), as was the internal control gene, G3PDH (primers listed above). The primer sequences and PCR conditions are as follows:

ACC metastasis-associated RNH gene 5'-ACTGAGATACCTACAGCGTG-3' and 5'-AGCGGTATCAGCTCACTCAA-3', $94^{\circ} \mathrm{C}$ for $3 \mathrm{~min}$, followed by 30 cycles of $94^{\circ} \mathrm{C}$ for $30 \mathrm{~s} ; 56^{\circ} \mathrm{C}$ for $45 \mathrm{~s} ; 72^{\circ} \mathrm{C}$ for $150 \mathrm{~s}$.

ACC metastasis-associated suspected protein gene 5'-ACGGCAGTTGTCAGATGTT-3' and 5'-CTTCTTCTACACTGCGTACACTG- 3 ', $94^{\circ} \mathrm{C}$ for $3 \mathrm{~min}$, followed by 30 cycles of $94^{\circ} \mathrm{C}$ for $30 \mathrm{~s} ; 57^{\circ} \mathrm{C}$ for $45 \mathrm{~s} ; 72^{\circ} \mathrm{C}$ for $150 \mathrm{~s}$.

G3PDH gene-primers listed above, $94^{\circ} \mathrm{C}$ for $3 \mathrm{~min}$, followed by 30 cycles of $94^{\circ} \mathrm{C}$ for $30 \mathrm{~s} ; 60^{\circ} \mathrm{C}$ for 45 s; $72{ }^{\circ} \mathrm{C}$ for $150 \mathrm{~s}$.

PCR products were separated by electrophoresis in a $1.5 \%$ agarose gel.

\section{Results}

\section{Ligation efficiency}

The G3PDH 3' primer and PCR primer 1 were used to amplify housekeeping gene G3PDH (glyceraldehyde-3-phosphate dehydrogenase) as an internal control, and the G3PDH $5^{\prime}$ and $3^{\prime}$ primers were used to

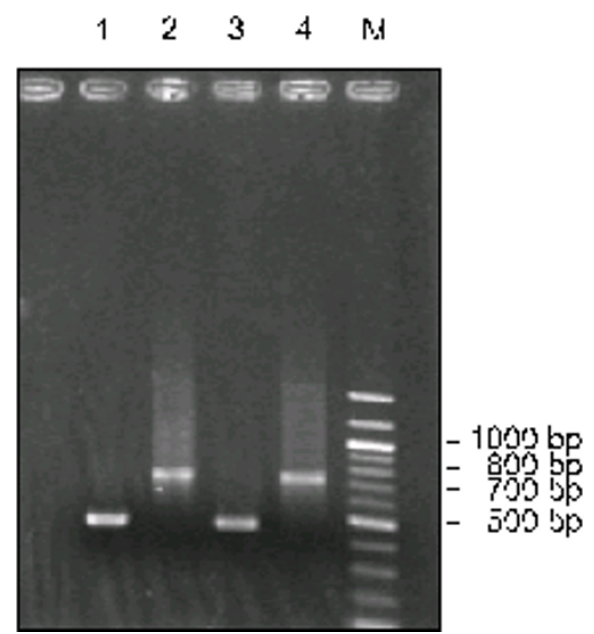

Figure 1. Typical results of ligation efficency analysis. Lane 1 shows PCR product using adaptor1-ligated tester as template, G3PDH $3^{\prime}$ primer \& PCR primer1, lane 2: PCR product using adaptor1-ligated tester as template, G3PDH 3' primer \& G3PDH 5' primer, lane 3: PCR product using adaptor2-ligated tester as template, G3PDH $3^{\prime}$ primer \& PCR primer1 and lane 4: PCR product using adaptor2-ligated tester as template, G3PDH 3' primer \& G3PDH 5' primer. M lane shows standard DNA molecular marker DL2000. amplify G3PDH with an adapter. The eletrophoretogram showed that the luminance difference was less than four-fold between PCR products, indicating a ligation efficiency within the required range (Figure 1). Different cycles of PCR were performed on the PCR secondary product with G3PDH $5^{\prime}$ and $3^{\prime}$ primers, and subtraction efficiency was measured and found to be within the required range.

\section{Differentially expressed genes and relevant homologous comparisons}

In this experiment, parallel suppressive subtractive hybridizations were performed using Acc-M as tester and Acc-2 as driver and vice versa. We were clearly able to visualize differences in the subtractive and non-subtractive products on agarose gels (Figure 2). Differentially expressed gene sequences were obtained using Acc-2 as driver, but none were identified when Acc-M was used as driver. After cloning and transformation, 12 differentially expressed genes were identified by DNA sequencing (Table 1). Of these, 10 gene fragments corresponded to known genes or sequences. Of these, the $G$ protein and ferritin I heavy chain genes were found to have mutations in their recovered partial nucleotide sequences (GenBank accession numbers were AF522027 and AF522026 respectively).

\section{Two novel ACC metastasis-associated gene fragments}

After cloning and sequence analysis of the differentially expressed cDNAs, we found that two of the clones had less than $80 \%$ homology with the registered gene sequences in GenBank. These new genes have been registered in GenBank (AF522024 and AF522025) as ACC metastasis-associated RNH (ribonuclease/angiogenin inhibitor) and ACC metasta-

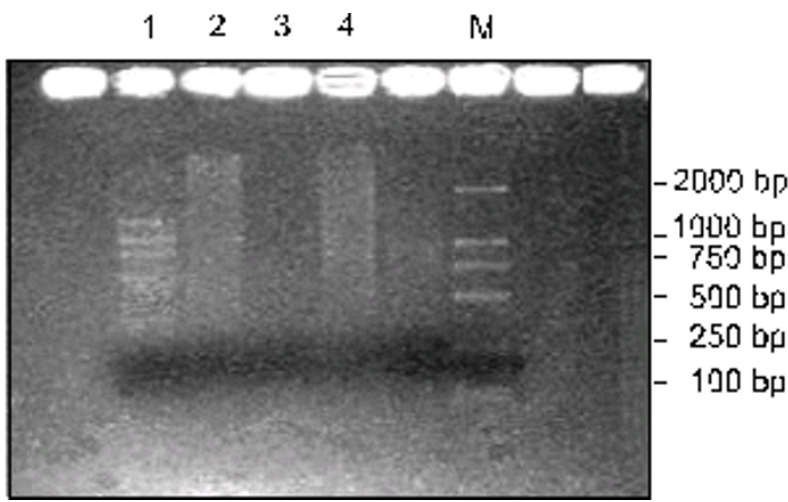

Figure 2. Typical results of subtraction experiment. Lane 1 shows subtracted product using Acc-2 as tester, lane 2: unsubtracted product using Acc-2 as tester, lane3: subtracted product using Acc-M as tester and lane 4: unsubtracted product using Acc-M as tester, M lane shows standard molecular marker DL2000. 
Table 1. Genes differentially expressed between the hiah and low metastasis cell lines.

\begin{tabular}{|c|c|c|c|c|}
\hline Clones & Genetic homologv and/or name & k accession & Function & Similar clones \\
\hline $\mathrm{C} 1$ & Cysteine-rich angiogenic-inducer protein & BC016952 & angiogenic-inducer & $\mathrm{n}=2$ \\
\hline $\mathrm{C} 2$ & Chromosome 7 clone RP11-52501 & AC021218 & Retinocytoma associated & $\mathrm{n}=2$ \\
\hline $\mathrm{C} 3$ & ACC metastasis-associated $G$ protein & AF522027\# & Cell signaling & $\mathrm{n} \geq 5$ \\
\hline $\mathrm{C} 4$ & ACC metastasis-associated heavy polypeptide I & AF522026\# & Metabolism & $n=3$ \\
\hline $\mathrm{C} 5$ & Jumping translocation breakpoint & BC019277 & DNA replication & $\mathrm{n}=1$ \\
\hline $\mathrm{C} 6$ & Eukaryotic translation elongation & BC012819 & Protein synthesis & $\mathrm{n}=2$ \\
\hline $\mathrm{C} 7$ & Folate receptor & BC002947 & Metabolism & $\mathrm{n}=3$ \\
\hline C8 & Human acidic ribosomal phosphoprotein PO mRNA & AK001313 & Protein synthesis & $\mathrm{n}=3$ \\
\hline $\mathrm{Cg}$ & ACC metastasis-associated RNH & AF522024* & ACC metastasis-associated & $\mathrm{n}=3$ \\
\hline C10 & Homo sapiens ribosomal protein L7a (RPL7A) & NM_000972 & Protein synthesis & $\mathrm{n}=4$ \\
\hline C11 & Homo sapiens ribosomal protein $\mathrm{S} 21$ & NM_001024 & Protein synthesis & $\mathrm{n}=1$ \\
\hline $\mathrm{C} 12$ & ACC metastasis-associated suspected protein & AF522025* & ACC metastasis-associated & $\mathrm{n}=1$ \\
\hline
\end{tabular}

Note: *represents the novel sequences; \#represents mutated sequences submitted by our laboratory.

A

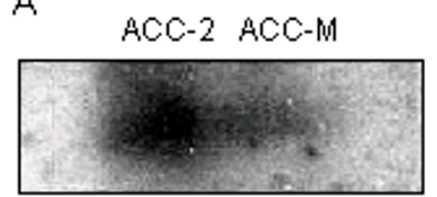

$\mathrm{B}$

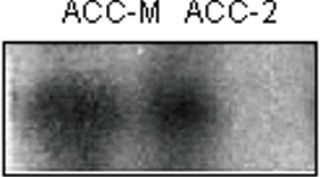

Figure 3. Representative Northern-Blot-analysis of ACC metastasisassociated RNH. A: Probed with ACC metastasis-associated RNH oligonucleotide 3'-labeled with DIG; B: Control hybridization was performed with G3'PDH probe. Total RNA $(6 \mu \mathrm{g})$ of ACC-M and ACC-2 cells was loaded onto each lane.

sis-associated suspected protein, respectively. These genes are expressed more highly in low metastasis cell line Acc-2 than in high metastasis cell line Acc-M.

\section{Northern blot and RT-PCR analysis}

Northern blot analysis showed that the ACC metastasis-associated RNH gene was expressed at low levels in AccM cells and at higher levels expressed in Acc2 cells. There was a 3-fold difference in expression level between the two cell lines (Figure 3 ). RT-PCR analysis confirmed expression of this gene in AccM cells, in accordance with the Northern blot results (Figure 4). We were unable to detect transcripts for ACC metastasis-associated suspected protein in either cell type by Northern blot analysis, perhaps because it is expressed at very low levels. However, RT-PCR analysis revealed the presence of this transcript in both cell types, although the expression level was more than 5 -fold lower in AccM cells than in Acc2 cells.
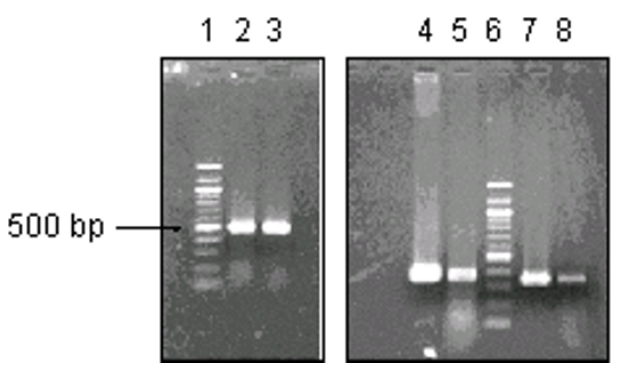

Figure 4. The RT-PCR results of ACC metastasis-associated RNH, ACC metastasis-associated suspected protein and G3PDH genes. Five microliter RT-PCR product were loaded in $1 \%$ agrose gel electrophoresis for each sample. Lane $1 \& 6$ shows 100 bp standard DNA marker Biolab), Lane 2 shows G3PDH RT-PCR product using AccM RNA as template, lane 3: G3PDH RT-PCR product using Acc2 RNA as template, lane 4: ACC metastasis-associated RNH RT-PCR product using Acc2 RNA as template, lane 5: ACC metastasis-associated RNH RT-PCR product using AccM RNA as template, lane 7: ACC metastasis-associated suspected protein RT-PCR product using Acc2 RNA as template, and lane 8: ACC metastasis-associated suspected protein RT-PCR product using AccM RNA as template.

\section{Discussion}

Tumor metastasis is regarded as the result of abnormal expression or inactivation of genes through a series of molecular changes in both tumor and host. Unrestricted growth and metastasis are the basic characteristics of cancer, which makes the study of tumor metastasis an important aspect of cancer research. So far there is no comprehensive theory that fully explains cancer metastasis and infiltration. Currently, it is thought that there are four characteristics of tumor metastasis: angiogenesis, binding, proteolysis and migration. That means that investigating one 
or two genes is not enough to understand the molecular mechanism of metastasis, and that cancer genetic research must embrace the dynamic variation of whole genome expression in order to understand the correlations genotype and phenotype.

Accordingly, we compared the high metastasis cell line of adenoid cystic carcinoma Acc-M with its original low metastasis mother cell line Acc-2, searching for differentially expressed genes that might be involved in the difference in metastasis. The result of this experiment shows that Acc-M contained 12 genes that were expressed at lower levels than those found in parent line Acc-2. Ten of these genes are known: cysteine-rich angiogenic-inducer protein, cyr61, RP11$52501, \mathrm{G}$ protein, ferritin, jumping translocation breakpoint (JTB), eukaryotic translation elongation factor, folate receptor and three ribosomal proteins.

Of these, cyr61 is a secretory cysteine-rich heparin binding protein that belongs to connective tissue growth factor family (CTGF) and is induced by growth factor TGF- $\beta$. Through an interaction with integrin, it causes endotheliocytes to bind and activates the proteins that communicate with paracrine cells. It was involved in the coordinated expression of growth factors in cytopoiesis and cellular differentiation and is an angiogenic-inducer (Grotendorst et al., 1997). Important in the cancer realm is the coordinated expression of cyr61 with c-fos and c-myc oncogenes (Lau et al., 1987). As a signal transfer protein, $G$ protein is known to be important in the invasion and binding of tumor cells. Previous tests have shown that high metastasis B16 mouse melanocyte cell line highly expressed $\mathrm{Gi} 2$ (one of the $\mathrm{G}$ proteins) in a pertussis toxin (PTX) sensitive manner. After treatment with PTX, ADP ribosylation occurred in $\mathrm{Gi}$, which significantly reduced the invasive ability of the tumor cells. Low metastasis B16 cell did not express Gi2, and the original characteristics (Lester et al., 1989) of the tumor cell remained unchanged after treatment with PTX.

We also found down-regulation in the high metastasis cell line of eukaryotic translation elongation, human acidic ribosomal phosphoprotein, several other ribosomal proteins and the metabolically relevant proteins ferritin and folate receptor. This probably reflects the increased metabolic needs of a tumor cell, and is supported by previous findings of association between low expression of ferritin and acquisition of metastasis (Jacqueline et al., 1997), and high expression of ribosomal proteins $\mathrm{L} 7 \mathrm{a}$ and $\mathrm{S} 21$ and human acidic ribosomal phosphoprotein in tumor cells (Zhang et al., 1997).

The jumping translocation breakpoint gene encodes a section of transmembrane protein that has been associated with the translocation of gene fragments within and between chromosomes. Tumor cells are known to be genetically characterized by chromosomal changes including addition, reduction, deletion or translocation. Several reports have discussed tumor suppressor sites and their inactivation through genetic disarray (Sekido et al., 1998; Petersen et al., $1997 ; 2000)$. A comparative genome hybridization of adenoid cystic carcinoma cell lines has shown characteristic duplications in 22q12.3-q13.1, and chromosomes 16 and 20 (Toida et al., 2001). Of the genes we found to be down-regulated, eukaryotic translocation elongation is located at $6 \mathrm{q} 14.1$, folate receptor at $11 q 13.3-14.1$, JTB at $1 \mathrm{q} 21$, RPS21 at $20 \mathrm{q} 13.3$, and cysteine-rich angiogenic-inducer protein (cyr61) at 1p31-p22. These results are in accordance with several of the putative tumor suppressor sites previously reported, and may have functional relevance in cancers other than Acc.

Also important in the study of metastatic genes is our finding of mutations in the gene fragments encoding $G$ protein and ferritin I heavy chain, suggesting that changes in these genes may be of oncogenic significance. Previous work has shown that the expression of ferritin heavy chain partial gene sequences can suppress the metastasis of a tumor (Jacqueline et al., 1997). These results, plus the two novel genes identified in this study, will bear further work to fully elucidate the roles of various genes in the metastasis of Acc tumors. Because of the heterogeneity of tumors and the complexity of the genetic changes inherent in malignancies, it is important to better understand the genes that undergo changes related to metastatic ability. Here, we report twelve such genes that are down-regulated in the high metastasis adenoid cystic carcinoma cell line Acc-M and discuss their possible roles as metastasis suppressors. This work provides a valuable set of biological materials and several new targets for further metastatic research, as well as possible new targets for tumor suppression therapy.

\section{Acknowledgement}

This work was supported by the national sciences foundation of China (No.30070816). The paper was proofread by a native English professional with science background at Elixigen Corporation.

\section{References}

Fang WG. Opportunity and challenge of research of tumor invasion and metastasis in $21^{\text {st }}$. Chin Medical J 2001;81: 193-4

Grotendorst GR. Connective tissue growth factor: a Mediator of TGF- $\beta$ Action on Fibroblasts. Cytokine \& Growth Factor Reviews 1997:8:171-9 
Guan X, Wang Y, Ge S, Li R. The comparative study on karyotypes of clones from salivary adenoid cystic carcinoma with high and low metastasis. J China Medical University 1996;25:368-70

Guan X, Qu W, He R, He RG, Zhou XJ. Selection of adenoid cystic carcinoma cell clone highly metastatic to the lung: an experimental study. International J Oral \& Maxillofacial Surgery 1997(a);26:116-9

Guan X, Li R, Wang Y. The inhibiting effect of RA against ACC-M clone metastatic to the lung. Oral \& Maxillofacial Surg J 1997(b);7:181-2

Guo J, Tao S, Zhou J, Zhuang J, Xie J, Luo C. Analysis of colon cancer related genes by suppression substractive hybridization. Chinese J Cancer 2001;20:743-7

Jacqueline AMV, Reindert JA, Van Moorselaar. Differential expression of ferritin Heavy chain in a rat transitional cell carcinoma progression model. Biochimica et Biophysica Acta 1997;(1360):39-44

Lau LF, Nathans D. Expression of a set of growth-related immediate early gene in BALB/C 3 T3 cells: coordinate regulation with c-fos or c-myc. Proc Natl Acad Sci USA 1987; 84:1182-6

Lester BR, McCarthy JB, Sun ZQ, Smith RS, Furcht LT, Spiegel AM. G-protein involvement in matrix-mediated motility and invasion of high and low experimental metastatic B16 melanoma clones. Cancer Res 1989;1;49:5940-8
Petersen I, Bujard M, Peterson S, Wolf G, Goeze A, Schwendel $A$, Langreck $H$, et al. Patterns of chromosomal imbalances in adenocarcinoma and squamous-cell carcinoma of the lung. Cancer Res 1997;57:2331-5

Petersen S, Aninat-Meyer M, Schlvns K, Dietel PI. Chromosomal alterations in the clonal evolution to the metastatic stage of squamous-cell carcinomas of the lung. Brit $\mathrm{J}$ Cancer 2000;82:65-73

Sekido $Y$, Fong KM, Minna JD. Progress in understanding the molecular pathogenesis of human lung cancer. Biochim Biophys Acta 1998;1378:F21-F59

Toida M, Balazs M, Mori T, Ishimaru JI, Ichihara H, Fujitsuka $\mathrm{H}$, Hyodo I, et al. Analysis of genetic alterations in salivary gland tumors by comparative genomic hybridization. Cancer Genetics and Cytogenetics 2001;127:34-7

VonStein OD, Thies WG, Hofmann M. A high throughput screening for transcribed differentially expressed genes. Nucleic Acids Res 1997;25:2598-602

Zhang L, Zhou W, Velculescu VE, Kern SE, Hruban RH, Hamilton SR, Vogelstein B, Kinzler KW. Gene expression profiles in normal and cancer cells. Science 1997;276:126872 